\title{
IDENTIFICACIÓN DE PROGRAMAS EDUCATIVOS PARA ADULTOS MAYORES, EN UNIVERSIDADES DE MEDELLÍN - COLOMBIA
}

\section{IDENTIFICATION OF EDUCATIONAL PROGRAMS FOR OLDER ADULTS, IN UNIVERSITIES OF MEDELLIN - COLOMBIA}

Nora Hincapié Gil nora.hincapie@salazaryherrera.edu.co Institución Universitaria Salazar Herrera Medellín- Antioquia

Fotografia de portada y Diagramación Sindy Catherine Charcas Ibarra 


\section{RESUMEN}

Objetivo. Identificar las universidades que imparten programas educativos para adultos mayores por extensión académica en Medellín, dando respuesta a la proyección comunitaria y la responsabilidad social que deben asegurar las instituciones de educación superior según la Ley 30 de 1992 (Colombia), posibilitando la participación de este grupo poblacional en actividades académicas y de bienestar universitario.

Metodología. El trabajo investigativo se orienta bajo el paradigma cualitativo, el enfoque interpretativo-comprensivo, mediante un estado del arte, que visibiliza los programas educativos en las universidades de Medellín, que además del componente académico propician espacios intergeneracionales y de inclusión social; permitiendo el desarrollo personal, familiar y comunitario, que promueven la cultura del envejecimiento con mejor calidad de vida.

Resultados. Como resultados se registran los siguientes programas: universidad Eafit "Saberes de vida" (Manjarres, 200I), universidad de Antioquia "Aula universitaria para mayores" (Zea, 2006), Tecnológico de Antioquia "Cátedra universitaria para adultos mayores" (Hincapié, 20l I), que surge de un proyecto de investigación, la Institución universitaria Salazar Herrera "Cátedra de la experiencia y la familia" (Hincapié, 20I6) y la Universidad Pontificia Bolivariana "Casa de la Felicidad" (Pelaez, 20I8). Estos programas se imparten con lineamientos del Modelo Gerontagógico.

Conclusión. De acuerdo a los índices estadísticos que evidencian el aumento de la población adulta mayor y la necesidad percibida en el medio de implementar programas y servicios, para la nueva generación de mayores; más activos, productivos, trayectoria académica, laboral y la participación en un mundo cambiante y en constante avance tecnológico, científico y médico que propone nuevos programas para esta población.

Palabras clave: programas educativos, adultos mayores, modelo gerontagógico, extensión académica, universidad.

\section{ABSTRACT}

Objective. To Identify the universities that provide educational programs focused on older adults through academic extension in Medellin, responding to community projection as a social responsibility which, must be guaranteed by higher education institutions according to Ley 30 de 1992 (Colombia), making possible the participation of this population group in academic activities and university welfare.

Methodology. This research project is based on a qualitative paradigm. The interpretative-comprehensive approach, through a state of the art, which makes visible the educational programs in the universities of Medellín, besides the academic component gives intergenerational and social inclusion spaces; allowing people personal, family and community development, encourage culture of aging for better quality life.

Results. The following programs are registered as a result:

Eafit University "Saberes de vida" (Manjarres, 200I),

Antioquia University "Aula Universitaria para Mayores" (Zea, 2006),

Tecnológico de Antioquia "Cátedra universitaria para adultos mayores" (Hincapié, 20II), which arises from a research project, Institución Universitaria Salazar Herrera "Cátedra de la experiencia y la familia" (Hincapié, 2016)

Pontificia Bolivariana University “Casa de la Felicidad” (Pelaez, 2018). These programs are taught with guidelines of the Gerontological Model.

Inferences. According to the statistical indexes that show the increase in the older population and the perceived insufficiency in the environment to implement programs and services for the new generation of older adults; more active, productive, academic, labor career and participation in a changing world and in a constant technological, scientific and medical progress that proposes new programs for this population.

Keywords: educational programs, older adults, gerontological model, academic extension, university. 


\section{INTRODUCCIÓN}

De acuerdo a las tendencias de la población adulta mayor actual; con mayor expectativa de vida, más activos, productivos y con deseos de participación y a la poca oferta de programas a nivel académico para este grupo poblacional que según los índices estadísticos evidencian el cambio de la pirámide poblacional, aumentando cada vez más; se crea la necesidad de implementar propuestas académicas, acordes al contexto actual que además del fortalecimiento intelectual, propicie espacios intergeneracionales y de inclusión social, como lo dice la ONU: "una sociedad para todas las edades. (1992).

Por lo anterior se hace necesario identificar los programas educativos para adultos mayores por extensión académica en las universidades de Medellín, bajo el modelo Gerontagógico, que tiene en cuenta sus condiciones a partir de las fortalezas adquiridas a través del tiempo de acuerdo a la experiencia personal, laboral y académica; permitiendo continuar la vida de forma activa, productiva, en los entornos universitarios.

Las nuevas generaciones de adultos mayores obtienen una mejor calidad de vida, invitando a reflexionar sobre los retos que se deben asumir en la oferta de programas y servicios que procuren una nueva construcción social y satisfagan los requerimientos de esta población. Según el médico especialista, Diego Bernardini:

Asistimos a una nueva vejez, una nueva etapa de vida; hoy aparece la nueva longevidad y esta nueva "etapa", que es una construcción social como las otras dos (niñez y adolescencia), condiciona nuevas necesidades, nuevas capacidades, nuevos mercados o nuevos desafíos (...) hoy las personas mayores son una generación más educada y eso les permite informarse, conocer, modificar hábitos de vida y, por, sobre todo, desafiar los cánones establecidos. (Bernardini, 2017).

La esperanza de vida, cada vez es más prolongada y las nuevas generaciones de adultos mayores vienen más activos, productivos y con deseos de participación; cada día se visibiliza mayor participación de este grupo poblacional en actividades culturales, artísticas, en los gimnasios, las universidades, con más vida nocturna y en programas intergeneracionales y de inclusión social. Como dice Bernardini "No solo hay más personas mayores, sino que la vida se extendió y hoy vivimos más años y de manera mucho más saludable, como nunca antes se había vivido en la historia de la humanidad".

\section{Estado del arte y antecedentes prácticos}

En los últimos años se ha evidenciado la intención que tienen las universidades en ofertar programas para todas las edades. Tras la búsqueda de información sobre estas experiencias se hacen hallazgos significativos sobre los diferentes programas educativos para adultos mayores, en varios países del mundo, que permiten vislumbrar la viabilidad de propuestas de inclusión social e intervención socioeducativa. La primera experiencia de programas educativos para personas mayores, tuvo su origen en Toulouse (Francia) con la primera
Universidad de la Tercera Edad, creada por el profesor Pierre Vellas en 1973, con algunos objetivos como:

Abrir una universidad para los retirados y facilitarles el acceso a la cultural de la humanidad, para la contribución a la prevención del declive psicosociológico y a la investigación científica sobre la vejez y formar a la población mayor para su reinserción social y participación comunitaria. (Vellas, 1995, s.p).

Desde que inició la primera universidad de la tercera edad, se han implementado este tipo de programas en muchas universidades de Europa y América del Norte, en los últimos años se han ido implementado en los países en vía de desarrollo en algunas universidades, rompiendo con los esquemas de la educación formal y ofertando programas de extensión académica donde solo se necesita voluntad y experiencia de vida para matricularse en los diplomados y además de lo académico disfrutan de los procesos intergeneracionales y de inclusión social, bajo distintos nombres: Programas Universitarios para Adultos Mayores, Aulas de la Experiencia, Universidades para la $3^{\mathrm{a}}$ Edad, Universidades Abiertas, y algunas asociaciones como la Red Iberoamericana de Asociaciones de Adultos Mayores (RIAAM), La Asociación Internacional de Universidades para la Tercera Edad y la Red de Universidades Abiertas, como referentes a las tendencias de una nueva modalidad en programas para esta población.

Se acoge la población adulta mayor dentro del campus universitario, ofertando programas de extensión académica - educación continua, buscando además del componente educativo tener inmersa esta población en procesos socioculturales intergeneracionales en procura de mejorar su calidad de vida y participación comunitaria.

\section{Marco teórico y referentes conceptuales}

En elámbito internacional, el Boletín de Envejecimiento de la Organización Mundial de la Salud (OMS) de 2012 da a conocer: "El mundo envejece y América Latina envejece más rápidamente que el viejo continente" y Colombia no es la excepción, por ello las instituciones del Estado, la academia, la sociedad civil y las personas en particular desde la primera infancia hasta los centenarios se deben preparar para asumir este fenómeno demográfico en el que toda la población y el planeta está involucrado, una de las alternativas enfatiza en la posibilidad de continuar la educación en otras condiciones a la escolarizada.

En Colombia la ley 125 I de 2008 del Congreso de la República, dicta normas tendientes a procurar la protección, promoción y defensa de los derechos de los adultos mayores, en el artículo 17. Áreas de intervención en cuanto a educación, cultura y recreación, reza: La educación, la cultura y la recreación hacen parte del proceso de formación integral del ser humano; con tal fin, el Estado deberá: (...) h) Proponer el acceso del adulto mayor a la educación formal e informal en diversas formas y niveles de capacitación a fin de lograr su desarrollo individual, familiar y social como forma de inclusión a la sociedad:

Colombia vive actualmente un proceso de transición demográfica plena, en el cual ha influido el descenso en la mortalidad, la paulatina 
disminución de la fecundidad y también las migraciones, la esperanza promedio de vida al nacer, cercana a los 48 años en 1950, fue de 74 años en el 2010" (DANE, 2010; Profamilia, 2010), "por lo demás, la población mayor de 60 años ha pasado de, aproximadamente el $6 \%$ en 1975 , a algo más del $10 \%$ en el 2012 y se prevé que para el 2050 será superior al $20 \%$ " (DANE, 2010), "La población mayor de 60 años en Colombia, ha aumentado a un ritmo superior al $3 \%$, lo cual contrasta con el ritmo de crecimiento de la población total, cercano al I,5 \%. (Naciones Unidas, 2002, p. 185). Según datos de la Dirección Seccional de Salud y Protección Social de Antioquia-DSSA, entre 1993 y 2005 la población de adultos mayores en Medellín creció un 6, 17\%. Es decir, de 165.704 personas mayores (9.2\% de la población) pasó a 258.937 (I I.7\%). A esto se le suma que en las cifras del Departamento Administrativo Nacional de Estadística- DANE se proyecta que para el 2015 de los más de 2.342.47I habitantes de Medellín la población de personas mayores será del I5.4\%. (Delaurbe, U de A. 20I3).

Debido al aumento de la esperanza de vida, se eleva considerablemente el número de personas mayores, con unas características que definen una nueva generación de mayores, debido a la mejor calidad de vida; siendo más activos, productivos y con deseos de participación en procesos educativos y socioculturales:

El proceso de envejecimiento está inminente en el ser humano, ligado al estilo de vida que se haya llevado en los años anteriores. La disminución de la fuerza y de la flexibilidad, la reducción de la longitud del paso de la marcha y una motricidad desequilibrada marcan el estrecho paso de la adultez a la adultez mayor. Acciones sistémicas a lo largo de la vida predeterminaran la clase de adulto mayor que se quiere ser; algunas de ellas beneficiaran la salud física y mental, como la actividad fisica regular, la buena alimentación y la estabilidad emocional, entre otras; estas forjaran un preciado y digno futuro en la etapa del adulto mayor (Álvarez y Alud 2017).

Las tendencias actuales de envejecimiento y vejez, invitan a reflexionar sobre los programas y servicios que existen para adultos mayores y la necesidad percibida en el medio de implementar propuestas de acuerdo a las nuevas características de este grupo poblacional, como enuncia Machersi: "El énfasis por tanto se sitúa en las políticas globales, capaces de plantear estrategias convergentes en las esferas económica, social y educativa con el objetivo de avanzar en la construcción de sociedades justas, cohesionadas y democráticas" (2012, p.9).

En la actualidad se presenta un gran reto para dar respuesta a las nuevas demandas educativas que surgen en los diferentes contextos, debido a los procesos sociales culturales de la globalización y los cambios que se evidencian con el avance tecnológico, científico y médico, propiciando al individuo trascender en el desarrollo humano integral competente para interactuar en la comunidad:

Según diferentes teorías filosóficas, el ser humano nace individuo y por la educación, llega a ser persona: La confianza, la aceptación del otro, el respeto, la acogida y la autenticidad son las nuevas actitudes de la relación educativa humanista que persigue, como objetivo educativo, su desarrollo armónico, para conseguir que la persona realice todos los valores positivos con la debida jerarquía entre ellos, surge la educación integral que ha de asegurar el perfeccionamiento de todos los aspectos de la personalidad: físicos, intelectuales, morales, afectivos, sociales, sexuales (Quintana, 1995, s.p).

La educación para toda la vida, favorece la inclusión de los diferentes grupos poblacionales en procesos de enseñanza aprendizaje, culturales y recreativos; recuperando saberes personales, actualizando conocimientos y participando en espacios académicos, que contribuyan a vislumbrar nuevos programas de intervención para las nuevas generaciones que están llegando en su mayoría profesionales; conllevando a establecer propuestas y lineamientos que favorecen este grupo poblacional.

De igual manera en las prácticas educativas se reconoce el aprendizaje para toda la vida, en correspondencia con el ciclo vital del adulto mayor; que según Rios "La educación permanente se ha convertido en la herramienta más necesaria para luchar contra las desigualdades, las injusticias y las situaciones que privan a las personas de vivir libre y dignamente en su medio" (1998. p.295).

Haciendo un análisis de adultos mayores de épocas anteriores y las generaciones actuales, en Colombia se percibe la necesidad de implementar programas educativos en las distintas universidades que permitan satisfacer los requerimientos académicos culturales de la comunidad en general, como compromiso de responsabilidad universitaria y proyección social. Según Renquejo “Entre las funciones tradicionales que competen hoy a las universidades asociadas al progreso y la transmisión del saber están: investigación, innovación, enseñanza y formación, educación permanente. A ellas se añade la cooperación internacional que cada vez cobra mayor importancia." (1999, p. I I2).

Las universidades deben estar preparadas para asumir los nuevos retos que demande la sociedad actual procurando una educación permanente que promueva la inclusión y vinculación de todos los grupos poblacionales, fortaleciendo procesos académicos investigativos que conllevan a establecer políticas públicas en beneficio de este grupo poblacional que se inscribe en los objetivos de la Agenda 2030 para el desarrollo sostenible: "Garantizando una vida sana, promoviendo el bienestar para todas las edades, propiciando oportunidades de aprendizaje e inclusión social, durante toda la vida para todos":

Para asegurar esta continuidad se insiste mucho en la creación de universidades para la tercera edad o instituciones similares. Lo que proponemos es que la comunidad requiere, de un lado, el mejoramiento de la calidad de la educación que se recibe en las escuelas, colegios y universidades. De otro, requiere que estas instituciones se abran a personas y grupos de tercera edad como estudiantes (reconociendo sus particularidades) y profesores voluntarios (Londoño, I99I.p. I28, 129).

Los programas educativos para adultos mayores comenzaron a tomar fuerza a partir de la prolongación en la expectativa de vida, las mejores condiciones de los adultos mayores a nivel de salud y la necesidad que se creó en las personas jubiladas, cuando se cuestionaron acerca del quehacer para seguir activos e inmersos en una sociedad productiva y participativa. Aunque la formación académica sigue teniendo la idea de formación profesional; la educación para adultos mayores se plantea como programa de extensión académica que permite el disfrute y la socialización del conocimiento, sin necesidad del rigor académico del estudiante de educación 
formal:

Para lograr la igualdad, evitar la discriminación por razón de edad, para universalizar el derecho a la educación: permitir la educación a lo largo de todo el ciclo vital. Para optimizar la eficacia y eficiencia de los recursos dedicados a los servicios que disfrutan los mayores (sociales, sanitarios, socio sanitarios, culturales, educativos etc.) (Bermejo, 2004. P.35).

Es importante construir procesos educativos y culturales participativamente, con las normas y orientaciones institucionales y el deseo y las propuestas del adulto mayor, dando como resultado un proceso educativo adecuado a los momentos de participación de los sujetos como protagonistas de la acción educativa y cuya intencionalidad procure no solo a remediar sino a potenciar los procesos de una práctica que combina la contribución real de las personas mayores a la apertura de espacios para incrementar su participación como individuos y como grupo social organizado.

Educar supone hacer dos trayectos, uno hacia sí mismo y otro hacia el entorno; quien asume una verdadera educación acepta la transformación personal y la social, las implicaciones educativas no pueden quedar aisladas, la educación de personas mayores no carece de un valor social; los mayores pueden ser productivos integralmente, aun admitiendo que el sentido de esta expresión no responde a las demandas de una sociedad inmersa en la rentabilidad del producto sobre los procesos que se ponen en marcha.

Se podría plantear que el objetivo de la educación es entendida como la posibilidad de llegar hacer audible, es decir posibilitar a la población mundial procesos educativos desde el inicio hasta el final de la vida; como estrategia para estar inmersos en los conocimientos de enseñanza-aprendizaje a través de toda la historia del ser humano. Para Machersi, "El sentido de la educación se encuentra y se reencuentra en la propia experiencia, en la conversación tranquila, en la reflexión personal sobre lo que cada uno vive y siente en la acción educadora" (2006, p.68).

Hablar de una educación para todas las edades, implica entrever que no solamente es útil en las primeras etapas de la vida, sino hasta el final de los días, pensar en ello es interpelar por la idea de que los aprendizajes realizados a través y durante toda la vida, posibilita enriquecer y construir la persona a través de los tiempos; en tanto esto es permitido y aceptable en la mente del ser humano y conlleva a pensar que los aprendizajes adquiridos se pueden colocar en la vejez al servicio de la construcción de sí mismo y las proyecciones a las comunidades más inmediatas:

Un artículo (...) publicado por el New York Times mostraba que, en Estados Unidos, mucha gente jubilada se muda cerca de las universidades para tener acceso a clases y a las bibliotecas. Ellos han tomado conciencia de que estudiar sirve, en primer lugar, para mantener intacta la integridad de las personas. Por esto, la educación a lo largo de la vida es fundamental (Machado, 2006, p. I29).

El desafío hoy se centra en la búsqueda del sentido de educación para todas las edades y equitativa, dando respuesta a las nuevas demandas educativas, procurando trabajar las desigualdades sociales y económicas, proyectando una sociedad que forje seres humanos socialmente responsables creativos y transformadores, una educación que enseñe a pensar y a proponer, que aliente a la construcción del conocimiento científico y la capacidad para el desarrollo tecnológico y la participación democrática. Coll afirma: "explorar la tesis de que los retos educativos fundamentales que enfrentamos en la actualidad provienen, al menos en parte, del desvanecimiento del sentido que ha tenido tradicionalmente la educación" (20I2, p.IOI).

Acorde a los planteamientos anteriores y bajo los auspicios y recomendaciones de los organismos mundiales de la salud, surgieron movimientos culturales que tratan de incluirlos, entre ellos la educación permanente para adultos mayores, que bajo el lema de Viguera, "se puede aprender durante toda la vida", (1997, s.p), abre lugares y lucha contra uno de los prejuicios muy arraigados todavía en el imaginario social que condiciona a la sociedad y a los mismos adultos mayores; dando como verdadero y universal el concepto referido a la pérdida de la posibilidad de aprender en el envejecimiento.

A partir de las diferentes experiencias de procesos educativos con la población adulta mayor, algunos autores fundamentan teorías de enseñanza aprendizaje dando origen a un modelo (todavía en construcción) denominado, "Modelo gerontagógico", considerado como el arte y la ciencia de enseñar a la población adulta mayor, según las posibilidades y capacidades actuales de aprendizaje y desarrollo de las nuevas generaciones de adultos mayores en la medida que surgen los programas educativos para este grupo poblacional, entre los referentes teóricos sobre el modelo gerontagógico, se destacan:

-La Gerontagogía como el ámbito científico y profesional, dedicado a la intervención socio-educativa con y para personas mayores, además pretende generar una atención específica con la intención de educar en el aprovechamiento máximo de las capacidades y experiencias que han ido acumulando los mayores a lo largo de su vida y que hoy en día suelen pasar desapercibidas en nuestra sociedad (Lemieux, 1997, p. 35).

-La Gerontagogía, según el profesor Lemieux, se define como una disciplina educativa interdisciplinar que tiene por objeto el estudio de la persona mayor en situación educativa. Etimológicamente provine del griego "gerontagogeo", que viene a significar "conducir a un viejo". (...) "Lo mismo que la pedagogía (ciencia aplicada) tiene como base teórica la psicología educativa, la gerontagogía tiene como base teórica la gerontología educativa” (Lemieux, 1997, p. 46).

- La educación de personas mayores aparecía no como una prolongación del modelo de formación inicial de los niños, ni como la reproducción de modelo de educación profesional y reciclaje/perfeccionamiento más propio de los jóvenes y adultos - aunque podía haber mayores necesidades de estos tipos de formación- Los programas educativos para mayores aportaban, como novedoso, una especifidad: "no se trata de desarrollar conocimientos nuevos, científicos. El modelo competencial trata sobre todo de reactualizar los conocimientos de cara a una mejor gestión de la vida personal y social. Aquí la persona no adquiere la ciencia, sino que aprende a servirse de ella dentro del principio de contradicción y de relatividad de todas las cosas, a lo que nosotros nos referimos como Sabiduría. Desde esta perspectiva, el 
conocimiento no se articula sobre el objeto de la Ciencia sino sobre la metacognición de la persona frente al objeto de su conocimiento" (Lemieux, 1998, p.220).

-En los últimos años se ha introducido en el lenguaje técnico-académico hispano el concepto de gerontagogía. Este concepto designa un campo de prácticas, discursos y saberes acerca de la educación de las personas mayores. Proclama para sí un recorte disciplinar que se ocupa de la reflexión pedagógica acerca de los gerontes (Yuny y Urbano, 2005, p.29).

-El termino gerontagogía pretende obtener un reconocimiento universal, lo que revela un intento unificador y totalizador de los discursos educativos que tematizan la educación de los mayores. Este tipo de discurso remite nuevamente a la tradición de las teorías educativas universales, propias de proyecto pedagógico moderno (...) La gerontagogía debe reconocer que su desarrollo supone una multiplicidad de perspectivas a cerca de la educabilidad de las personas mayores sobre los valores pedagógicos que sustentan su teleología (Yuny y Urbano, 2005, p.3I).

-La Gerontagogía como ciencia que estudia el hecho y las posibilidades educativas en las personas mayores y plantea cuatro itinerarios que ilustran especialmente los espacios de la educación en el último tramo vital; los estudios de la Gerontagogía señalan unos mapas por los que puede navegar la persona entrada en años a condición de una disponibilidad biológica, mental y son: La cultura y la creatividad, el escenario de la naturaleza, el ocio como desarrollo festivo y escenario del compromiso social (García, 2004, P.I I8).

En la propuesta educativa gerontagógica, como práctica social sustenta el piso teórico, hacía una visión más creíble, transformadora y posibilitadora de la educación como proceso que interviene en el ser humano en toda sus dimensiones, tanto físicas, psicológicas, sociales y culturales:

Es fundamental que la metodología sea activa, participativa, socializadora, indagadora en la medida de lo posible y sin duda, abierta y comprensiva con el fin primordial de facilitar oportunidades de recrear la vida del mayor, dándole una nueva significación a su trayectoria vital (Blázquez, 2000, p. II).

La función mediadora de los programas educativos en los entornos universitarios, evidencian el desarrollo de una cultura de inclusión socioeducativa, que asumen todos los grupos generacionales como una riqueza, constituyéndose en una base sólida para lograr aprendizajes integrales que generen mayores niveles de satisfacción en toda la población y concibiendo a las personas como seres activos, con deseos de participar en actividades de: estimulación cognitiva, lúdicas, académicas y culturales; reconociendo una estrategia idónea para ayudar en la prevención del deterioro de las facultades mentales, para mejorar la calidad de vida, como menciona Freire en su tratado de Pedagogía de la Autonomía: "Programados para aprender e imposibilitados de vivir sin la referencia de un mañana, donde quiera que haya mujeres y hombres habrá siempre qué hacer, habrá siempre qué enseñar, habrá siempre qué aprender" (1997, s.p).

La Gerontagogía fomenta una mayor calidad de vida porque concibe que la planificación y puesta en marcha de programas culturales $y$ educativos puede $y$ debe tender a la autorrealización deseada propiciando una visión transformadora en el ser humano a través de todas sus dimensiones: físicas, psicológicas, sociales y culturales, "Desde una perspectiva evolutiva trata además de enfatizar y destacar una imagen más positiva de los mayores a través de una designación específica lo que permite revitalizar más que encubrir otros ámbitos relacionados como el de la Pedagogía Social” (Sáez, 2003, p. 50).

En el ámbito mundial se visibilizan diferentes experiencias investigativas que dan cuenta de los avances de las propuestas educativas para adultos mayores, y entre ellas están:

La Red Iberoamericana de Programas Universitarios para Adultos Mayores (RIPUAM) está constituida por universidades públicas y privadas de España y América Latina que cuentan entre sus fines, con la formación universitaria para mayores, se trata de una asociación de interés universitario cuyo fin esencial es fomentar los programas educativos para mayores en el ámbito universitario (Bru, 20l I, p.2).

Como referente de estas experiencias; España es uno de los países que más registran programas educativos para adultos mayores en las universidades, en Cuba las cátedras universitarias del adulto mayor, constituyen un proyecto atendido por la dirección de Extensión Universitaria perteneciente al sistema nacional de educación y cumple a su vez uno de los objetivos de la Nueva Universidad Cubana, es por ello que constituye una parte de la batalla de ideas que libran el país; "no se reproduce ninguna carrera universitaria, sino que tiene como misión la superación cultural y la actualización científico-técnica de las personas mayores” (García, 2006, s.p).

En América del sur el país que más registra programas educativos para adultos mayores dentro de las universidades es Argentina, seguida de Chile, Perú, Brasil, y Costa Rica. En Colombia la mayoría de programas se registran en la ciudad de Medellín.

La implementación de programas educativos para adultos mayores en algunas universidades de Medellín (Institución Universitaria: Tecnológico de Antioquia y Salazar y Herrera, surgen de un proceso investigativo fundamentado en: el "Proyecto piloto de implementación de, "Universidad para Adultos Mayores como programa de extensión académica" (Hincapié, 20I I, p.74I).

Cada vez se evidencia más, la implementación de programas educativos para adultos mayores en las universidades, por lo anterior se realiza un rastreo de los programas educativos y sus objetivos, en algunas universidades de Medellín, referenciado los siguientes programas:

- $\quad$ SABERES DE VIDA - Universidad Eafit: Surge de la idea de crear un espacio donde los adultos pudieran continuar enriqueciéndose intelectualmente, un espacio donde se pudiera aprender sin el estrés de las evaluaciones, un espacio donde los estudiantes encontraran un "BIEN-ESTAR", un espacio donde pudieran lograrse PROYECCIONES individuales y grupales, un espacio donde compartir con pares generacionales... Saberes de Vida es un espacio para vivir la vida universitaria. (Manjarrés, M. 200I). 
AULA UNIVERSITARIA PARA MAYORES Universidad de Antioquia: El Aula Universitaria de Mayores surge como respuesta a las necesidades educativas de los adultos mayores de Medellín y su área metropolitana, desde la perspectiva vida-salud-enfermedad. Se plantean los principales aspectos conceptuales y de la experiencia de un programa que durante casi 3 años de trayectoria ha permitido la participación de más de 120 personas adultas mayores, con buena evaluación y aceptación. Objetivo: presentar algunas reflexiones conceptuales y asuntos relevantes de la experiencia del Aula Universitaria de Mayores en la Facultad de Enfermería de la Universidad de Antioquia. Conclusiones: los adultos mayores conservan repertorios para defenderse en la vida cotidiana; su educación constituye un reto para los profesionales y amerita concebirse como necesidad, derecho y servicio público (Zea, 2009. P. 244-252).

\section{CÁTEDRA UNIVERSITARIA PARA ADULTOS} MAYORES -Tecnológico de Antioquia: Surge de un proyecto de investigación: Proyecto piloto de implementación de universidad para adultos mayores en Medellín Colombia, y posteriormente implementado como "Cátedra universitaria para adultos mayores" en el Tecnológico de Antioquia, avalado por el Consejo Académico, según resolución 05 del 29 de noviembre de $201 \mathrm{I}$, como programa de extensión académica, en un entorno universitario que además del componente académico propicia espacios intergeneracionales y de inclusión social en busca de una mejor calidad de vida de este grupo poblacional (Hincapié, 20I2).

- $\quad$ CÁTEDRA DE LA EXPERIENCIA Y LA FAMILIA Institución Universitaria Salazar y Herrera: La Cátedra de la Experiencia y la Familia de la organización Salazar y Herrera, surge por la necesidad sentida en el medio de establecer programas y servicios para este grupo poblacional en las diferentes sedes, con una intención humanizada, que se inscribe en los objetivos de la Agenda 2030 para el desarrollo sostenible: "Garantizando una vida sana, promoviendo el bienestar para todas las edades, propiciando oportunidades de aprendizaje e inclusión social, durante toda la vida para todos" y bajo el ideal filosófico institucional proyectado en la persona, la familia y la sociedad (Hincapié, 2016).

- $\quad$ CASA DE LA FELICIDAD - Universidad Pontificia Bolivariana: Casa de la Felicidad es un programa de formación cognitiva, espiritual, artística y de oficios cuyo objetivo es brindar espacios de aprendizaje y crecimiento personal en el disfrute, la socialización, la apropiación social del conocimiento y la generación de comunidad como contexto propicio de bienestar, interacción y goce para el adulto. Es el camino para la construcción de una morada interior en la que se aprende a vivir, a convivir y a trascender; mediante el equilibrio consigo mismo, con los otros, con el mundo y con Dios. Casa de la Felicidad es el camino para la realización humana, para vivir en alegría, para disfrutar y cultivar el mundo interior (Pelaez, 2018).

\section{Metodología}

La investigación se orienta bajo el paradigma cualitativo, el enfoque interpretativo-comprensivo, mediante la realización de un estado del arte, que posibilita visibilizar las características de los programas educativos para adultos mayores en algunas universidades de Medellín: Universidad Eafit con el programa: Saberes de vida (200I), la facultad de enfermería de la Universidad de Antioquia con Zea: Aula para mayores (2009), el Tecnológico de Antioquia con "Hincapié: La Cátedra Universitaria para Adultos Mayores" que surge de un proyecto de investigación (20I2) y la Institución Universitaria Salazar y Herrera con la "Cátedra de la Experiencia y la Familia" (2016) y la Universidad Pontificia Bolivariana "Casa de la Felicidad" (Pelaez, 20I8).

Entre sus características están: ser adulto mayor participante en programas académicos; estudiantes mayores de 50 años, en condiciones físico funcionales y psicológicas normales para interactuar en este tipo de programas. La investigación utiliza como técnicas la entrevista semiestructurada para conocer las expectativas de los adultos mayores que participan en los programas, antes de la aplicación del instrumento se obtiene la autorización del consentimiento informado para las personas que acceden a la entrevista, la utilización de los datos y la publicación de resultados, con reserva de nombres de las personas entrevistadas, adicional, la autorización por escrito de las instituciones que ofertan los programas definidos en la muestra.

\section{Análisis y resultados}

La identificación de los programas educativos para adultos mayores en universidades de Medellín, permite visibilizar la proyección y el impacto social que generan estas universidades en sus comunidades, permitiendo procesos intergeneracionales de inclusión muy similares, bajo los lineamientos del modelo Gerontagógico que permite evidenciar la flexibilidad, el compartir conocimiento, la no evaluación cuantitativa, y el fortalecimiento de las redes de apoyo. Los adultos mayores que participan en programas educativos, obtienen un reconocimiento social como personas activas, comprometidas en procesos que inciden de manera efectiva en el progreso de la sociedad, permitiendo ser visibilizados como adultos mayores que continúa en busca del desarrollo individual, familiar y comunitario; y estos a su vez tienen la posibilidad de estar, más de cerca con el interactuar de los jóvenes en los distintos entornos universitarios. La autoevaluación realizada en los procesos educativos para adultos mayores, como seguimiento para la cualificación de los programas; ya que no se realiza evaluación formal, posibilita en el enfoque Gerontagógico el reconocimiento positivo de sí mismo y el bienestar personal.

La Intergeneracionalidad surge como factor relevante en los procesos educativos en los entornos universitarios, ya que permiten el encuentro de estos dos grupos generacionales (estudiantes de pregrado y estudiantes adultos mayores) impactando en los espacios universitarios como propuesta de inclusión social, que empiezan a implementarse en algunas universidades de la ciudad. La participación en los programas educativos, fomenta el desarrollo en dimensiones individuales, familiares y sociales, permitiendo al adulto mayor hacer un reconocimiento de sus virtudes, saberes y cualidades que los lleva a vincularse en este tipo de programas, fortaleciendo su 
proceso intelectual, aumentando la autoestima, identificando una mayor autonomía y capacidad para la toma de sus decisiones, la satisfacción y el bienestar personal que se refleja en la participación; ya que la gente está cautivada y generalmente es muy cumplida con la asistencia, se estudia más que todo por disfrutar la academia.

El autorreconocimiento que adquieren en los procesos educativos es una de los resultados más relevantes en esta investigación ya que se evidencia una gran satisfacción de los adultos mayores por la dinámica de este tipo de programas; ya que según expresan: "nos estamos dando un regalo con estos diplomados". Los adultos mayores que participan en los programas educativos, adquieren un reconocimiento social, ya que son visibilizados como personas activas y productivas con deseos de continuar en procesos académicos y de inclusión social, con deseos de superación y participando en procesos que inciden de manera efectiva en el progreso de la sociedad. Uno de los grandes investigadores en esta temática, expresa: García, J. "escenario del compromiso social; entendiéndose por compromiso social aquella proyección que hace de sus miembros sujetos partícipes de una acción comunitaria, en este apartado se hace referencia al desarrollo comunitario, el voluntariado y el asociacionismo" (2004, p. I I8).

Desde que se inició la primera universidad de la tercera edad en Toulouse, Francia (1973) se han implementado este tipo de programas en muchas universidades de Europa y América del Norte, en los últimos años se han ido implementado en los países en vía de desarrollo este tipo de programas en algunas universidades, rompiendo con los esquemas de la educación formal y ofertando programas de extensión académica donde solo se necesita voluntad y experiencia de vida para matricularse en los diplomados y además de lo académico disfrutan de los procesos intergeneracionales y de inclusión social. Los espacios universitarios, propician un encuentro con otros grupos poblacionales como: adultos mayores, estudiantes, empleados y docentes de la universidad; permitiendo evidenciar las características y formas de interactuar de los distintos grupos etarios, y según expresan "se está con la juventud y aunque no se hable directamente con ellos al menos se ven interactuar y ellos nos ven a nosotros estudiando," es una ventaja que da el campo universitario.

El reconocimiento de los aprendizajes adquiridos a través de la experiencia de vida, en relación a los nuevos aprendizajes que están recibiendo en su ciclo vital; actualizando, reajustando, reconstruyendo, compartiendo y socializando de una forma libre, espontánea, donde eligen las temáticas y áreas de conocimiento que les interesa profundizar, sin ninguna presión de tareas y evaluaciones en este proceso educativo y que según expresan "además del interés por algunas temáticas, se percibe la oportunidad de seguir en procesos académicos, ya que es una forma de ampliar pensamientos, permitiendo estar mentalmente abierto y pensando, recibiendo conocimiento para seguir reflexionando, "el adulto mayor no es como el niño que está en procesos educativos y no se da cuenta que está aprendiendo; el adulto mayor goza cuando ve que está aprendiendo algo y disfruta el aprendizaje".

La comprensión del aprendizaje en el ciclo de vida de los adultos mayores, trasciende la postura de vejez como estado estático; por el estado de envejecimiento activo, productivo, con deseos de continuar participando, además contribuye al ámbito de la investigación; ya que ratifica el interés que tiene este grupo poblacional en continuar los procesos educativos a lo largo de la vida; que además del componente académico les propicie: enriquecimiento personal, participación social y encuentros intergeneracionales. Se puede afirmar que el aprendizaje es significativo en esta etapa de la vida, por el sentido que tiene para el crecimiento personal; es un conocimiento que se adquiere para fortalecerse interiormente y procurar una mejor calidad de vida, ya que se está en la edad donde se alcanza el autorreconocimiento de muchos valores adquiridos a través de la vida, y como lo expresan: "toda la vida se la entregue a mi familia y mi trabajo, este es el momento para encontrarme a mí misma y aprender cosas que me enriquezcan y me den satisfacción personal".

\section{Conclusiones}

- La identificación de programas educativos para adultos mayores en universidades de Medellín, permiten además del componente académico, propiciar espacios de inclusión social, dentro del campus universitario, dando respuesta a la proyección comunitaria y la responsabilidad social que deben asegurar las instituciones de educación superior según la Ley 30 de 1992 (Colombia) y a las recomendaciones de las Naciones Unidas: "Hacia una sociedad para todas las edades".

Los programas universitarios para adultos mayores, por sus objetivos y estructura organizativa, contribuyen en la creación de una sociedad inclusiva, donde las acciones educativas muestran cómo la universidad, a la que asisten jóvenes y mayores de edad, constituye el marco idóneo para las relaciones intergeneracionales

- La educación para adultos mayores por extensión académica es abierta, flexible y establece cambios de buenas prácticas académicas, permitiendo la visibilidad en contextos de inclusión social, participando en entornos intergeneracionales, como parte del desarrollo psicosocial, sin pensar en estudiar por una nota o para salir a laborar.

En los procesos educativos dirigidos a personas mayores se adquieren comparten y actualizan conocimientos, propiciando escenarios académicos y encuentros de saberes; los docentes orientan a estudiantes con experiencias de vida laborales y profesionales que enriquecen y reconstruyen las temáticas propuestas en el aula.

- $\quad$ Si la propuesta educativa se puede implementar en otras universidades, $y$ tiene continuidad en el tiempo; se ha contribuido en procesos de transformación, en programas para adultos mayores, creyendo que el envejecimiento es un acto de vida; es decir una acción educativa. 


\section{Referencias bibliográficas}

Agenda 2030 para el Desarrollo Sostenible (2015). Objetivos de Desarrollo Sotenible.

Álvarez, E. \& Alud, A. (2017). La actividad física y sus beneficios físicos como estrategia de inclusión social del adulto mayor. Revista de inclusión \& Desarrollo, 5 (I), 23-36.

Bermejo, L. (2004). Gerontología Educativa. Como diseñar proyectos educativos con personas mayores. Editorial Panamericana, Madrid.

Bernardini, D. (2017). De qué hablamos, cuando hablamos de "Nueva longevidad". Argentina.

Blázquez, F. (2000). Memorias del Programa Universidad de los Mayores de Extremadura. Revista Badajoz: ECE de la UEX.

Bru, C. (20II). La Universidad también es para mayores. Revista, Universidad de Alicante, España.

Congreso de la República de Colombia. (1992). Ley 30: por el cual se organiza el servicio público de la Educación Superior.

Congreso de la República de Colombia. (2008). Ley I25I: Por la cual se dictan normas tendientes a procurar la protección, promoción y defensa de los derechos de los adultos mayores.

Coll, C. (20I2). Enseñar y aprender en el siglo XXI: El sentido de los aprendizajes escolares. Revista Redalyc.org.

Dane. (20I0). Encuesta Nacional de Demografía y salud - ENDS. Colombia.

Delaurbe. (2013). En Medellín cada vez hay más personas mayores.

Facultad de comunicaciones, Universidad de Antioquia.

Fernández, C. (2002). "La nueva filosofía de integrar a los mayores en la universidad”. Sevilla, España

Freire, P. (1997). A la sombra de este árbol. Barcelona: Roure.

García, J. (2004). La educación en personas mayores. Ensayo de nuevos caminos. Narcea S. A. Ediciones. Madrid, España.

Hincapié, N. (20I I). Proyecto piloto de implementación de Universidad para Adultos mayores como programa de extensión académica en el Tecnológico de Antioquia. IV Congreso Iberoamericano de Universidades para Adultos Mayores. Asociación Estatal de Programas Universitarios para Mayores (AEPUM). Volumen II. Alicante, España.

Hincapié, N. (2016). Cátedra de la Experiencia y la Familia del Salazar y Herrera, Primer Congreso Internacional, Profesionalización del Docente Universitario. Universidad Autónoma Latinoamericana. Medellín, Colombia.

Lemieux, A. (1997). Los programas universitarios para mayores. Enseñanza E investigación. IMSERSO. Madrid, España.

Londoño, L. (199I). Gerontología Educativa. Medellín - Colombia

Manjarres, M.V. (200I). Programa Educativo para Adultos Mayores: Saberes de 
Vida, Universidad Eafit. Medellín, Colombia.

Machado, A. (2006). Los Sentidos de la Educación, Por el humanismo en la Educación, Brasil. Revista Prelac, Volumen II.

Marchesi, A. (2006). Los Sentidos de la Educación, El valor de educar a todos en un mundo diverso y desigual, España. Revista Prelac, Volumen II.

Marchesi, A. (20I2). Calidad equidad y reformas en la enseñanza. Libro, fundación Santillana. España.

OMS (2012). Boletín de Envejecimiento de la Organización Mundial de la Salud

Peláez, B. (2018). Programa Educativo para Adultos Mayores: Casa de la Felicidad. Universidad Pontificia Bolivariana. Medellín, Colombia.

Quintana, J. (1995). Teoría de la Educación. Madrid: Dykinson

Renquejo, A. (1999). Educación de adultos: Programas universitarios para personas mayores. Revista de estudios y experiencias educativas. Santiago de Compostela, España.

RIPUAM (20I I). Red Iberoamericana de programas educativos para adultos mayores. Alicante, España.

Rios, F. (2003) El contenido de la educación permanente en las conferencias mundiales de la UNESCO. Revista Pedagogía Social Interuniversitaria, N. 10, p. $281-297$

Sáez, J. (2003). “Tercera edad, Necesidad de Educación y aprendizaje en las Personas mayores" Madrid: Editorial Dykinson.

Universidad de Vida y Esperanza. (2009). Antecedentes de la UNI 3. Monografías página web, Venezuela Recuperado de:

http://www.monografias.com/trabajos82/universidad-vida-y-esperanza/uni.v.

Vellas, P. (1995). Recherches et réalisations. Pedone. Paris.

Viguera, V. (1997). Educación para el envejecimiento - El portal de la psicogerontología.

Yuni, J. \& Urbano, C. (2005). Educación de adultos mayores. Teoría, investigación e intervenciones. Editorial Brujas, Córdoba, Argentina.

Zea, C. (2006). Programa Educativo para Adultos Mayores: Aula Universitaria para mayores, Universidad de Antioquia. Medellín, Colombia. 